\title{
Quadratic function fields whose class numbers are not divisible by three
}

\author{
by \\ Humio ICHimura (Yokohama)
}

1. Introduction. For an algebraic number field $K$, let $C l(K)$ be its ideal class group and $h(K)=|C l(K)|$. For a prime number $l$ dividing the degree $[K: \mathbb{Q}]$, we have a lot of information on the $l$-part $C l(K)(l)$ of $C l(K)$ (see e.g. [2], [3], [11], [14]). On the other hand, when $l \nmid[K: \mathbb{Q}]$, not so many results are known on $C l(K)(l)$. One of such is that of Hartung [8] and Horie [9], who proved that there exist infinitely many imaginary quadratic fields $K$ with $l \nmid h(K)$ (and satisfying some additional conditions) for any odd prime number $l$. When $l=3$, there are stronger results concerning the "density" of the set of quadratic fields $K$ with $3 \nmid h(K)$ (and satisfying some additional conditions), which were obtained by Davenport and Heilbronn [5], Datskovsky and Wright [4], and Kimura [12]. They also obtained analogous results for quadratic extensions over the rational function field $\mathbb{F}_{q}(T)$, where $\mathbb{F}_{q}$ is a fixed finite field.

Since the methods in the papers referred to above are not constructive, it is desirable to give explicit families of infinitely many quadratic extensions $K$ over $\mathbb{Q}$ or $\mathbb{F}_{q}(T)$ with $l \nmid h(K)$ for each odd prime number $l$. Here, $h(K)$ is the number of divisor classes of $K$ of degree zero when $K$ is a function field of one variable over a finite constant field. The main purpose of this note is to give such families when $l=3$ in the function field case.

Let us give the main results. Let $p$ be a fixed prime number, $q$ a fixed power of $p$, and $\mathbb{F}_{q}$ the finite field with cardinality $q$. Let $T$ be a fixed indeterminate. We take the rational function field $\mathbb{F}_{q}(T)$ as the base field. For simplicity, we assume $p \geq 5$ in this section. For $n \geq 1$ and $a \in \mathbb{F}_{q}^{\times}$, we put

$$
L_{n, a}=\mathbb{F}_{q}\left(T,\left(T^{3^{n}}+a\right)^{1 / 2}\right) .
$$

1991 Mathematics Subject Classification: Primary 11R58; Secondary 11R11, 11R29.

The author was partially supported by Grant-in-Aid for Scientific Research (C), Grant Number 11640041. 
The genus of $L_{n, a}$ is $\left(3^{n}-1\right) / 2$. We show that $3 \nmid h\left(L_{n, a}\right)$ when $q \equiv 1 \bmod 3$ and $a \notin\left(\mathbb{F}_{q}^{\times}\right)^{2}$ (Theorem $1(\mathrm{II})$ ). However, when $q \equiv-1 \bmod 3$, we have $3 \mid h\left(L_{n, a}\right)$ for all $a \in \mathbb{F}_{q}^{\times}$and $n$ (Theorem 1 (III)). So, we have to find another family. We define rational functions $X_{n}=X_{n}(T)$ in $\mathbb{F}_{q}(T)$ inductively as follows:

(1) $\quad X_{0}=T, \quad X_{n}=\left(X_{n-1}^{3}-3 X_{n-1}-1\right) /\left(3\left(X_{n-1}^{2}+X_{n-1}\right)\right)$ for $n \geq 1$.

We easily see that when $q \equiv-1 \bmod 3$, there exists $\gamma \in \mathbb{F}_{q}^{\times}$such that $\gamma^{2}-3 \gamma+9 \notin\left(\mathbb{F}_{q}^{\times}\right)^{2}$. We put

$$
L_{n}^{\prime \prime}=\mathbb{F}_{q}\left(T,\left(3 X_{n}+\gamma\right)^{1 / 2}\right) .
$$

The genus of $L_{n}^{\prime \prime}$ is $3^{n}-1$. We show that $3 \nmid h\left(L_{n}^{\prime \prime}\right)$ for all $n \geq 1$ when $q \equiv-1 \bmod 3$ (Theorem 4 ). We give similar families also when $p=2,3$ (Theorem 4, Theorem 3).

REMARK 1 . The second formula in (1) is a variant of the polynomial $f_{a}=X^{3}-a X^{2}-(a+3) X-1(a \in \mathbb{Z})$. This polynomial was first effectively used by Shanks [16]. A property of $f_{a}$ is that its discriminant is $\left(a^{2}+3 a+9\right)^{2}$, which is used in the proof of Theorem 4.

REMARK 2. Let $\infty_{T}$ be the prime divisor of $\mathbb{F}_{q}(T)$ corresponding to the pole of $T$. After Artin [1], we say that a quadratic extension $K / \mathbb{F}_{q}(T)$ of nonzero genus is a "real" quadratic extension when $\infty_{T}$ splits, and an "imaginary" one otherwise. The quadratic extensions given in Theorems 1-4 in Section 2 are imaginary ones.

Remark 3. Nagell [13] (resp. Yamamoto [17]) constructed infinitely many imaginary (resp. real) quadratic extensions (over $\mathbb{Q}$ ) whose class numbers are divisible by a given integer. For analogous results for the function field case, see Friesen [6] and the author [10].

Convention. For the rational function field $\mathbb{F}_{q}(X)$ with an indeterminate $X$, we denote by $\infty_{X}$ its prime divisor corresponding to the pole of $X$. Further, for an irreducible monic $P=P(X)$ in the polynomial ring $\mathbb{F}_{q}[X]$, we denote by $(P)$ the prime divisor of $\mathbb{F}_{q}(X)$ corresponding to the zeros of $P$. When $l \neq p$, let $\mu_{l \infty}$ be the group of $l^{a}$ th roots of unity for all $a \geq 1$ in the algebraic closure $\overline{\mathbb{F}}_{q}$, and $\zeta_{l^{a}}$ a primitive $l^{a}$ th root of unity. For a module $M$, we abbreviate the quotient $M / l M$ (or $M / M^{l}$ ) by $M / l$.

2. Families of quadratic extensions over $\mathbb{F}_{q}(T)$. Let $q$ be a fixed power of a prime number $p$, and $l$ a fixed odd prime number. In this section, we give several families of quadratic extensions $L$ over $\mathbb{F}_{q}(T)$ with $l \mid h(L)$ (resp. $l \nmid h(L)$ ). The results announced in Section 1 for $l=3$ are contained in these ones. 
For an element $x$ of the algebraic closure $\overline{\mathbb{F}_{q}(T)}$, we put

$$
x^{\mathcal{P}}=x^{p}-x \quad \text { and } \quad x^{\mathcal{P}^{n}}=\left(x^{\mathcal{P}^{n-1}}\right)^{\mathcal{P}} \quad \text { for } n \geq 1 .
$$

We also denote by $x^{1 / \mathcal{P}^{n}}$ an element $z$ satisfying $z^{\mathcal{P}^{n}}=x$.

First, assume that $l \neq p$. For $n \geq 1$ and $a \in \mathbb{F}_{q}$, we put

$$
L_{n, a}= \begin{cases}\mathbb{F}_{q}\left(T,\left(T^{l^{n}}+a\right)^{1 / 2}\right) & \text { for } p \neq 2, \\ \mathbb{F}_{q}\left(T,\left(T^{l^{n}}+a\right)^{1 / \mathcal{P}}\right) & \text { for } p=2 .\end{cases}
$$

Here, we assume $a \neq 0$ when $p \neq 2$. Let $\delta_{l}(q)$ be the order of $q \bmod l$ in the multiplicative group $(\mathbb{Z} / l \mathbb{Z})^{\times}$, and let $\mathbb{F}_{q}^{\mathcal{P}}$ be the subset of $\mathbb{F}_{q}$ consisting of elements $x^{\mathcal{P}}$ with $x \in \mathbb{F}_{q}$. For the quadratic extensions $L_{n, a}$, we prove the following assertions.

Theorem 1. Assume that $l \neq p$ and $p \neq 2$.

(I) When $a \in\left(\mathbb{F}_{q}^{\times}\right)^{2}$, we have $l \mid h\left(L_{n, a}\right)$ for all $n$.

(II) When $\delta_{l}(q)$ is odd, we have $l \mid h\left(L_{n, a}\right)$ if and only if $a \in\left(\mathbb{F}_{q}^{\times}\right)^{2}$.

(III) When $\delta_{l}(q)=2$, we have $l \mid h\left(L_{n, a}\right)$ for all $a$ and $n$.

Theorem 2. Assume that $l \neq p$ and $p=2$.

(I) When $a \in \mathbb{F}_{q}^{\mathcal{P}}$, we have $l \mid h\left(L_{n, a}\right)$ for all $n$.

(II) When $\delta_{l}(q)$ is odd, we have $l \mid h\left(L_{n, a}\right)$ if and only if $a \in \mathbb{F}_{q}^{\mathcal{P}}$.

(III) When $\delta_{l}(q)=2$, we have $l \mid h\left(L_{n, a}\right)$ for all $a$ and $n$.

Next, assume that $l=p$. For $n \geq 1$ and $a \in \mathbb{F}_{q}$, we put

$$
L_{n, a}^{\prime}=\mathbb{F}_{q}\left(T,\left(T^{\mathcal{P}^{n}}+a\right)^{1 / 2}\right) .
$$

For these quadratic extensions, we prove the following:

Theorem 3. Assume that $l=p$. We have $l \nmid h\left(L_{n, a}^{\prime}\right)$ for all $a$ and $n$.

Finally, let $l=3$ and $q \equiv-1 \bmod 3$. Let $X_{n}=X_{n}(T)$ be the rational function in $\mathbb{F}_{q}(T)$ defined by (1), and when $p \neq 2$, let $\gamma$ be a fixed element of $\mathbb{F}_{q}^{\times}$such that $\gamma^{2}-3 \gamma+9 \notin\left(\mathbb{F}_{q}^{\times}\right)^{2}$. For $n \geq 1$, we put

$$
L_{n}^{\prime \prime}= \begin{cases}\mathbb{F}_{q}\left(T,\left(3 X_{n}+\gamma\right)^{1 / 2}\right) & \text { for } p \neq 2 \\ \mathbb{F}_{q}\left(T,\left(X_{n}\right)^{1 / \mathcal{P}}\right) & \text { for } p=2\end{cases}
$$

For these quadratic extensions, we prove the following:

TheOREM 4. Assume that $l=3$ and $q \equiv-1 \bmod 3$. We have $3 \nmid h\left(L_{n}^{\prime \prime}\right)$ for all $n$.

REMARK 4 . When $\delta_{l}(q)$ is even but not 2 , the author could not show whether or not $l \mid h\left(L_{n, a}\right)$ for $a \notin\left(\mathbb{F}_{q}^{\times}\right)^{2}$.

3. Some lemmas. Let $k$ be a fixed algebraic function field of one variable with constant field $\mathbb{F}_{q}$, and let $l$ be a fixed prime number (not necessarily 
odd). In this section, we give several lemmas concerning the class number $h(k)$ of $k$ or that of a finite separable extension over $k$. They are well known or, otherwise, known to specialists.

The following lemma follows from class field theory.

LEMma 1. Let $\mathfrak{p}$ be a prime divisor of $k$ with $l \nmid \operatorname{deg}(\mathfrak{p})$, where $\operatorname{deg}(*)$ denotes the degree of a divisor. Then $l \mid h(k)$ if and only if there exists an unramified cyclic extension over $k$ of degree $l$ in which $\mathfrak{p}$ splits completely.

For this, the readers may consult Rosen [15, p. 368]. From this lemma, we immediately obtain the following corollaries.

Corollary 1 . Let $\mathfrak{p}$ be as in Lemma 1 . Let $\mathbb{F}_{Q} / \mathbb{F}_{q}$ be a finite extension and $K=k \mathbb{F}_{Q}$. Assume that $\mathfrak{p}$ remains prime in $K$. Then $l \mid h(K)$ if $l \mid h(k)$.

Corollary 2. Let $\mathfrak{p}$ be as in Lemma 1 . Let $K / k$ be a finite separable extension in which $\mathfrak{p}$ is totally ramified. Then $l \mid h(K)$ if $l \mid h(k)$.

The following lemma is a function field analogue of a theorem of Iwasawa [11] on the class numbers of algebraic number fields.

Lemma 2. Let $K / k$ be a finite $l$-Galois extension. Assume that exactly one prime divisor $\mathfrak{P}$ of $K$ is ramified over $k$ and that $l \nmid \operatorname{deg}(\mathfrak{P})$. Then $l \mid h(K)$ implies $l \mid h(k)$.

Proof. Though this assertion is more or less known, we give a proof for the convenience of the readers. Assume that $l \mid h(K)$. Let $H / K$ be the maximal unramified abelian extension of exponent $l$ in which $\mathfrak{P}$ splits completely. As $l \mid h(K)$, we have $H \neq K$ by Lemma 1 . Put $\mathfrak{p}=\mathfrak{P} \cap k$. Then we see that $\mathfrak{P}$ is the unique prime divisor of $K$ over $\mathfrak{p}$ from an assumption of the lemma. Therefore, $H$ is Galois over $k$. Let $G=\operatorname{Gal}(H / k)$ and $Z(\subseteq G)$ the decomposition group of an extension of $\mathfrak{P}$ in $H$. We have $G \neq Z$ as $H \neq K$. Then, since $G$ is an $l$-group, there exists a normal subgroup $\widetilde{Z}$ of $G$ such that $[G: \widetilde{Z}]=l$ and $\widetilde{Z} \supseteq Z$ (cf. Hall [7, Theorem 4.3.2]). Let $E$ be the intermediate field of $H / k$ corresponding to $\widetilde{Z}$ by Galois theory. Then $E / k$ is an unramified cyclic extension of degree $l$, and $\mathfrak{p}$ splits completely in $E$. Therefore, we obtain $l \mid h(k)$ by Lemma 1.

The following is a version of Lemma 2. As in Section 1, we denote by $\infty_{T}$ the prime divisor of $\mathbb{F}_{q}(T)$ corresponding to the pole of $T$.

Lemma 3. Let $k=\mathbb{F}_{q}(T)$ and $K / k$ a finite $l$-Galois extension. Assume that $q \equiv 1 \bmod l$. Assume further that (i) $\infty_{T}$ is totally ramified in $K$, (ii) exactly one prime divisor $\mathfrak{p}$ of $k$ other than $\infty_{T}$ is ramified in $K$, and (iii) $l \nmid \operatorname{deg}(\mathfrak{p})$. Then $l \nmid h(K)$.

Proof. Assume that $l \mid h(K)$. Then, in a way similar to the proof of Lemma 2 , we see that there exists a cyclic extension $E$ over $k$ of degree $l$ 
unramified outside $\mathfrak{p}$ in which $\infty_{T}$ splits completely. Let $P=P(T)\left(\in \mathbb{F}_{q}[T]\right)$ be the irreducible monic corresponding to $\mathfrak{p}$. Since $q \equiv 1 \bmod l$, we can write $E=\mathbb{F}_{q}\left(T,\left(\zeta P^{a}\right)^{1 / l}\right)$ for some $\zeta \in \mathbb{F}_{q}^{\times}$and $a \in \mathbb{Z}$. Then, since $l \nmid \operatorname{deg}(P)$ and $\infty_{T}$ splits in $E$, it follows that $l \mid a$ and $\zeta \in\left(\mathbb{F}_{q}^{\times}\right)^{l}$, and hence $E=k$. This is a contradiction.

The following lemma is known as Abhyankar's lemma (cf. Cornell [2]).

LEMMA 4. Let $E_{i}$ be a finite separable extension over a local field $\kappa$ with ramification index $e_{i}(i=1,2)$. If $E_{2}$ is at most tamely ramified and $e_{2} \mid e_{1}$, then $E_{1} E_{2} / E_{1}$ is unramified.

Finally, assume that $l \neq \operatorname{char}(k)(=p)$. Let $\zeta=\zeta_{l}$ be a primitive $l$ th root of unity, $K=k(\zeta)$ and $\Delta=\operatorname{Gal}(K / k)$. Let $\infty$ be a fixed prime divisor of $k$ such that $\operatorname{deg}(\infty)$ is relatively prime to $l|\Delta|$. There exists a unique prime divisor $\widetilde{\infty}$ of $K$ over $\infty$ as $\operatorname{deg}(\infty)$ and $|\Delta|$ are relatively prime. For $v \in K^{\times}$, we denote by $[v]$ the class in $K^{\times} / l=K^{\times} /\left(K^{\times}\right)^{l}$ represented by $v$. We regard $K^{\times} / l$ as a module over the group ring $\mathbb{F}_{l}[\Delta]$. For an $\mathbb{F}_{l}[\Delta]$-module $M$ and an $\left(\mathbb{F}_{l}\right.$-valued) character $\chi$ of $\Delta$, let $M(\chi)$ denote the $\chi$-component of $M$. Namely, $M(\chi)$ is the maximal submodule of $M$ on which $\Delta$ acts via $\chi$. Let $\omega$ be the $\left(\mathbb{F}_{l}\right.$-valued) character of $\Delta$ representing its Galois action on $\zeta$, and $\chi_{0}$ the trivial character of $\Delta$.

Lemma 5. In the above setting, we have $l \mid h(k)$ if and only if there exists a nontrivial element $[v]$ of $\left(K^{\times} / l\right)(\omega)$ or $\left(K^{\times} / l\right)\left(\chi_{0}\right)$ such that (i) the cyclic extension $K\left(v^{1 / l}\right) / K$ of degree $l$ is unramified and (ii) $\widetilde{\infty}$ splits completely in this extension.

Proof. Denote by $C l_{K}$ the divisor class group of $K$ of degree zero. Let $\widetilde{H} / K$ be the maximal unramified abelian extension of exponent $l$, and $H$ the maximal intermediate field of $\widetilde{H} / K$ in which $\widetilde{\infty}$ splits completely. The fields $\widetilde{H}$ and $H$ are Galois also over $k$ as $\widetilde{\infty}$ is the unique prime of $K$ over $\infty$. We put $A=\operatorname{Gal}(H / K)$. Further, let $\widetilde{V}$ and $V$ be the subgroups of $K^{\times} / l$ such that

$$
\widetilde{H}=K\left(v^{1 / l} \mid[v] \in \widetilde{V}\right) \quad \text { and } \quad H=K\left(v^{1 / l} \mid[v] \in V\right)
$$

respectively. The groups $A, \widetilde{V}, V$ as well as $C l_{K} / l=C l_{K} / C l_{K}^{l}$ are naturally regarded as modules over $\mathbb{F}_{l}[\Delta]$ since $\widetilde{H}$ and $H$ are Galois over $k$. By class field theory, we have a canonical isomorphism $C l_{K} / l \cong A$ compatible with the action of $\Delta$. So, we identify these two modules. We see that $l \mid h(k)$ if and only if $\left(C l_{K} / l\right)\left(\chi_{0}\right)$ is nontrivial from class field theory (cf. [15, p. 368]).

Now, let $\chi$ be any $\mathbb{F}_{l}$-valued character of $\Delta$. We prove the following:

Claim 1. The dimensions of the four vector spaces

over $\mathbb{F}_{l}$ are equal.

$$
\left(C l_{K} / l\right)(\chi), \quad\left(C l_{K} / l\right)\left(\omega \chi^{-1}\right), \quad V(\chi), \quad V\left(\omega \chi^{-1}\right)
$$


The desired assertion follows from this.

Let $\mu_{l^{a}}=\mu_{l^{\infty}} \cap K$. Then we easily see that $\widetilde{H}=H\left(\zeta_{l^{a+1}}\right)$. From this, it follows that

$$
\operatorname{dim} \widetilde{V}(\chi)= \begin{cases}\operatorname{dim} V(\chi) & \text { for } \chi \neq \omega \\ \operatorname{dim} V(\chi)+1 & \text { for } \chi=\omega\end{cases}
$$

Here, $\operatorname{dim}(*)$ denotes the dimension of $*$ over $\mathbb{F}_{l}$. For each element $[v] \in \widetilde{V}$, the principal divisor $(v)$ is written as $(v)=\mathfrak{A}^{l}$ for some divisor $\mathfrak{A}$ of $K$. By mapping $[v]$ to the divisor class $[\mathfrak{A}]$ of $\mathfrak{A}$, we obtain the following exact sequence:

$$
0 \rightarrow \mu_{l^{a}} / \mu_{l^{a-1}} \rightarrow \widetilde{V} \rightarrow{ }_{l} C l_{K} \rightarrow 0 .
$$

Here, ${ }_{l} C l_{K}$ is the elements $a$ of $C l_{K}$ with $a^{l}=1$. Clearly, this sequence is compatible with the $\Delta$-action. Hence, by (2), we obtain

$$
\operatorname{dim}\left(C l_{K} / l\right)(\chi)=\operatorname{dim}\left({ }_{l} C l_{K}\right)(\chi)=\operatorname{dim} V(\chi)
$$

for any $\chi$. On the other hand, the Kummer pairing

$$
A \times V \rightarrow \mu_{l}, \quad(\sigma,[v]) \rightarrow\langle\sigma,[v]\rangle=\left(v^{1 / l}\right)^{\sigma-1}
$$

is nondegenerate and satisfies

$$
\left\langle\sigma^{\varrho},[v]^{\varrho}\right\rangle=\langle\sigma,[v]\rangle^{\varrho}=\langle\sigma,[v]\rangle^{\omega(\varrho)} \quad \text { for } \varrho \in \Delta .
$$

From this, we easily obtain

$$
\operatorname{dim}\left(C l_{K} / l\right)(\chi)=\operatorname{dim} V\left(\omega \chi^{-1}\right)
$$

for any $\chi$. The assertion of Claim 1 follows from (3) and (4).

4. Proof of Theorems 1 and 2. We give a proof only for the case $p \neq 2$ (Theorem 1$)$. The case $p=2$ (Theorem 2) can be proved in a similar way.

We assume that $l \neq p$ and $p \neq 2$. We fix $a \in \mathbb{F}_{q}^{\times}$, and write $L_{n}=L_{n, a}$ for brevity. Putting $Y=\left(T^{l^{n}}+a\right)^{1 / 2}$, we have

$$
L_{n}=\mathbb{F}_{q}\left(Y,\left(Y^{2}-a\right)^{1 / l^{n}}\right) .
$$

Proof of (I) and (III). The prime divisor $\infty_{Y}$ of $\mathbb{F}_{q}(Y)$ is totally ramified in the extension $L_{n} / \mathbb{F}_{q}(Y)$. Therefore, we see that the condition $l \mid h\left(L_{n-1}\right)$ implies $l \mid h\left(L_{n}\right)$ by the second corollary of Lemma 1 . Hence, it suffices to prove the assertions (I) and (III) only when $n=1$. We write $L=$ $L_{1}$ for brevity. Let $\zeta=\zeta_{l}$, and let $Q=\left|\mathbb{F}_{q}(\zeta)\right|$ so that $\mathbb{F}_{Q}=\mathbb{F}_{q}(\zeta)$. Put $\widetilde{L}=$ $L \mathbb{F}_{Q}$. We identify the Galois group $\Delta=\operatorname{Gal}\left(\mathbb{F}_{Q} / \mathbb{F}_{q}\right)$ with $\operatorname{Gal}\left(\mathbb{F}_{Q}(Y) / \mathbb{F}_{q}(Y)\right)$ and $\operatorname{Gal}(\widetilde{L} / L)$ in the obvious way. Let $\widetilde{\infty}_{Y}$ be the unique prime divisor of $\widetilde{L}$ over $\infty_{Y}$.

First, assume that $a=b^{2}$ with $b \in \mathbb{F}_{q}^{\times}$. Put $v=(Y-b) /(Y+b)$. Clearly, we have $[v] \in\left(\widetilde{L}^{\times} / l\right)\left(\chi_{0}\right)$. We see that the cyclic extension $\widetilde{L}\left(v^{1 / l}\right) / \widetilde{L}$ is 
unramified by Lemma 4 , and that $\widetilde{\infty}_{Y}$ splits completely in this extension as $v \equiv 1 \bmod (1 / Y)$. Therefore, by Lemma 5 , we get $l \mid h(L)$.

Next, assume that $\delta_{l}(q)=2$ and $a \notin\left(\mathbb{F}_{q}^{\times}\right)^{2}$. The condition $\delta_{l}(q)=2$ implies $|\Delta|=\left[\mathbb{F}_{Q}: \mathbb{F}_{q}\right]=2$. Hence, $a=\alpha^{2}$ for some $\alpha \in \mathbb{F}_{Q}^{\times}$. Put $v=$ $(Y-\alpha) /(Y+\alpha)$. We have $[v] \in\left(\widetilde{L}^{\times} / l\right)(\omega)$ as $\delta_{l}(q)=2$. We see that the cyclic extension $\widetilde{L}\left(v^{1 / l}\right) / \widetilde{L}$ is unramified and that $\widetilde{\infty}_{Y}$ splits completely in this extension similarly to the above. Therefore, we get $l \mid h(L)$ by Lemma 5 . The assertions (I) and (III) follow from these.

Pro of of (II). By (I), it suffices to show that $l \nmid h\left(L_{n}\right)$ when $a \notin\left(\mathbb{F}_{q}^{\times}\right)^{2}$. So, we assume $a \notin\left(\mathbb{F}_{q}^{\times}\right)^{2}$. Let $Q_{n}=\left|\mathbb{F}_{q}\left(\zeta_{l^{n}}\right)\right|$ so that $\mathbb{F}_{Q_{n}}=\mathbb{F}_{q}\left(\zeta_{l^{n}}\right)$. We put $\widetilde{L}_{n}=L_{n} \mathbb{F}_{Q_{n}}$. To prove $l \nmid h\left(L_{n}\right)$, it suffices to show $l \nmid h\left(\widetilde{L}_{n}\right)$ because of the first corollary of Lemma 1 . As $\delta_{l}(q)=\left[\mathbb{F}_{Q_{1}}: \mathbb{F}_{q}\right]$ is odd, $\left[\mathbb{F}_{Q_{n}}: \mathbb{F}_{q}\right]$ is also odd. Hence, $a \notin\left(\mathbb{F}_{Q_{n}}^{\times}\right)^{2}$, and $Y^{2}-a$ is irreducible over $\mathbb{F}_{Q_{n}}$. Therefore, the extension $\widetilde{L}_{n}$ over $\mathbb{F}_{Q_{n}}(Y)$ satisfies the assumptions of Lemma 3 , and hence, we obtain $l \nmid h\left(\widetilde{L}_{n}\right)$.

5. Proof of Theorem 3. We assume that $l=p$. We fix $a \in \mathbb{F}_{q}$, and write $L_{n}^{\prime}=L_{n, a}^{\prime}(n \geq 1)$ for brevity. Putting $Y=\left(T^{\mathcal{P}^{n}}+a\right)^{1 / 2}$, we have

$$
L_{n}^{\prime}=\mathbb{F}_{q}\left(Y,\left(Y^{2}-a\right)^{1 / \mathcal{P}^{n}}\right) \quad(n \geq 1) .
$$

We put $L_{0}^{\prime}=\mathbb{F}_{q}(Y)$. Let $Z=\left(Y^{2}-a\right)^{1 / \mathcal{P}^{n-1}}$. Then

$$
L_{n-1}^{\prime}=\mathbb{F}_{q}(Y, Z) \quad \text { and } \quad L_{n}^{\prime}=\mathbb{F}_{q}\left(Y, Z^{1 / \mathcal{P}}\right) .
$$

The prime divisor $\infty_{Z}$ of $\mathbb{F}_{q}(Z)$ is ramified in the quadratic extension $L_{n-1}^{\prime} / \mathbb{F}_{q}(Z)$. The Artin-Schreier extension $\mathbb{F}_{q}\left(Z^{1 / \mathcal{P}}\right) / \mathbb{F}_{q}(Z)$ is unramified outside $\infty_{Z}$ and is totally ramified at $\infty_{Z}$. Therefore, we see that the cyclic extension $L_{n}^{\prime} / L_{n-1}^{\prime}$ of degree $l=p$ is ramified only at the unique prime of $L_{n-1}^{\prime}$ over $\infty_{Z}$. Then, by Lemma 2 , the condition $l \mid h\left(L_{n}^{\prime}\right)$ implies $l \mid h\left(L_{n-1}^{\prime}\right)$. From this, we obtain the assertion as $l \nmid h\left(L_{0}^{\prime}\right)$.

6. Proof of Theorem 4. We give a proof only for the case $p \neq 2$. The case $p=2$ can be proved in a similar way.

We assume that $l=3, q \equiv-1 \bmod 3$ and $p \neq 2$. Fix $n \geq 1$. For $1 \leq i \leq n$, we put

$$
N_{i}=\mathbb{F}_{q}\left(X_{n-i}\right) \quad \text { and } \quad M_{i}=\mathbb{F}_{q}\left(X_{n-i},\left(3 X_{n}+\gamma\right)^{1 / 2}\right) .
$$

Then we see from (1) that

$$
N_{1} \subseteq N_{2} \subseteq \ldots \subseteq N_{n}=\mathbb{F}_{q}(T), \quad M_{1} \subseteq M_{2} \subseteq \ldots \subseteq M_{n}=L_{n}^{\prime \prime}
$$


and that $M_{i} / N_{i}$ is a quadratic extension. The polynomial $P_{i}=X_{n-i}^{2}+$ $X_{n-i}+1$ in $\mathbb{F}_{q}\left[X_{n-i}\right]$ is irreducible as $q \equiv-1 \bmod 3$. We denote by $\left(P_{i}\right)$ the prime divisor of $N_{i}$ corresponding to the zeros of $P_{i}$.

To prove Theorem 4, we prepare several claims.

Claim 2. The extension $N_{i+1} / N_{i}$ is cyclic cubic and unramified outside $\left(P_{i}\right)$. We have $\left(P_{i}\right)=\left(P_{i+1}\right)^{3}$ in this extension.

Proof. Put $Y=X_{n-(i+1)}$ and $Z=X_{n-i}$ for brevity. Then $N_{i+1}=$ $\mathbb{F}_{q}(Y)$ and $N_{i}=\mathbb{F}_{q}(Z)$. By (1), $Y$ is a root of the polynomial $Y^{3}-3 Z Y^{2}-$ $3(1+Z) Y-1$ over $\mathbb{F}_{q}(Z)$. The discriminant of this polynomial is $3^{4}\left(Z^{2}+Z+1\right)^{2}$. Hence, $N_{i+1} / N_{i}$ is a cyclic cubic extension, in which $\left(P_{i}\right)$ is ramified. Since

$$
P_{i}=Z^{2}+Z+1=\left(Y^{2}+Y+1\right)^{3} /\left(9\left(Y^{2}+Y\right)^{2}\right),
$$

we see that $\left(P_{i}\right)=\left(P_{i+1}\right)^{3}$ in $N_{i+1}=\mathbb{F}_{q}(Y)$. Finally, we see that the other primes are unramified in $N_{i+1} / N_{i}$ because $N_{i}$ and $N_{i+1}$ are of genus zero and because of the Riemann-Hurwitz formula for genus of algebraic function fields.

Let $\zeta=\zeta_{3}$, and $\mathbb{F}_{Q}=\mathbb{F}_{q}(\zeta)$ with $Q=q^{2}$.

Claim 3. $\gamma+3 \zeta$ is not a square in $\mathbb{F}_{Q}^{\times}$.

Proof. Assume, on the contrary, that $\gamma+3 \zeta=(\lambda+\mu \zeta)^{2}$ for some $\lambda, \mu \in \mathbb{F}_{q}$. Clearly, $\mu \neq 0$. By the above, we get

$$
\gamma=\lambda^{2}-\mu^{2} \quad \text { and } \quad 3=2 \lambda \mu-\mu^{2} .
$$

From this, we obtain

$$
3(\lambda / \mu)^{2}-2 \gamma(\lambda / \mu)+(\gamma-3)=0 .
$$

Hence, the discriminant $4\left(\gamma^{2}-3 \gamma+9\right)$ of this quadratic polynomial must be a square in $\mathbb{F}_{q}^{\times}$. This contradicts the choice of $\gamma$.

Claim 4. The prime $\left(P_{1}\right)$ of $N_{1}$ remains prime in the quadratic extension $M_{1} / N_{1}$.

Proof. We see from (1) that

$$
3 X_{n}+\gamma \equiv 3 X_{n-1}+\gamma \bmod P_{1}\left(=X_{n-1}^{2}+X_{n-1}+1\right) .
$$

Since $\zeta$ is a root of $P_{1}$, the assertion follows from Claim 3 .

Claim 5. We have $3 \nmid h\left(M_{1}\right)$.

Proof. Put $Y=X_{n-1}$ and $Z=\left(3 X_{n}+\gamma\right)^{1 / 2}$. Then $M_{1}=\mathbb{F}_{q}(Y, Z)$. We see that the genus of $M_{1}$ is 2 because exactly 6 prime divisors are ramified in the quadratic extension $M_{1} \overline{\mathbb{F}}_{q} / \overline{\mathbb{F}}_{q}(Y)$. In the following, we view $M_{1}$ as an extension over $\mathbb{F}_{q}(Z)$. By (1), $Y$ is a root of the polynomial

$$
Y^{3}-\left(Z^{2}-\gamma\right) Y^{2}-\left(Z^{2}-\gamma+3\right) Y-1
$$


over $\mathbb{F}_{q}(Z)$. The discriminant of this polynomial is $P^{2}$ with

$$
P=P(Z)=\left(Z^{2}-\gamma\right)^{2}+3\left(Z^{2}-\gamma\right)+9
$$

A root $\alpha$ of $P(Z)$ satisfies $\alpha^{2}=\gamma+3 \zeta$. Then, by Claim 3, we see that $\alpha$ is of degree 4 over $\mathbb{F}_{q}$, and hence, $P$ is irreducible over $\mathbb{F}_{q}$. From the above, we see that $M_{1} / \mathbb{F}_{q}(Z)$ is a cyclic cubic extension, in which the prime of $\mathbb{F}_{q}(Z)$ corresponding to the irreducible monic $P(Z)$ is ramified. Since the genus of $M_{1}$ is 2 and $\operatorname{deg}(P)=4$, we see that the other primes of $\mathbb{F}_{q}(Z)$ are unramified in $M_{1}$ by the Riemann-Hurwitz formula. Hence, we obtain $3 \nmid h\left(M_{1}\right)$ by Lemma 2 .

Claim 6. Assume that $3 \nmid h\left(M_{i}\right)$ and the prime $\left(P_{i}\right)$ of $N_{i}$ remains prime in the quadratic extension $M_{i} / N_{i}$. Then we have $3 \nmid h\left(M_{i+1}\right)$, and $\left(P_{i+1}\right)$ remains prime in $M_{i+1} / N_{i+1}$.

Pr o of. Since $M_{i+1}=M_{i} N_{i+1}$, we obtain the assertion by using Claim 2 and Lemma 2.

Now, we obtain Theorem 4 for the case $p \neq 2$ from Claims 4,5 and 6 .

The case $p=2$ can be proved in a similar way by using, in place of Claim 3, the following:

Claim 7. Let $p=2$ and $q \equiv-1 \bmod 3$. Then $T^{4}+T+1$ is irreducible over $\mathbb{F}_{q}$.

\section{References}

[1] E. Artin, Quadratische Körper im Gebiet der höheren Kongruenzen I und II, Math. Z. 19 (1923), 153-246.

[2] G. Cornell, Abhyankar's lemma and the class group, in: Number Theory, Carbondale, 1979, M. Nathanson (ed.), Lecture Notes in Math. 751, Springer, New York, $1981,82-88$.

[3] - Relative genus theory and the class group of l-extensions, Trans. Amer. Math. Soc. 277 (1983), 321-429.

[4] B. Datskovsky and D. J. Wright, Density of discriminants of cubic extensions, J. Reine Angew. Math. 386 (1988), 116-138.

[5] H. Davenport and H. Heilbronn, On the density of discriminants of cubic fields II, Proc. Roy. Soc. London Ser. A 322 (1971), 405-420.

[6] C. Friesen, Class number divisibility in real quadratic function fields, Canad. Math. Bull. 35 (1992), 361-370.

[7] M. Hall, The Theory of Groups, Macmillan, New York, 1959.

[8] P. Hartung, Proof of the existence of infinitely many imaginary quadratic fields whose class numbers are not divisible by three, J. Number Theory 6 (1976), 276-278.

[9] K. Horie, A note on basic Iwasawa $\lambda$-invariants of imaginary quadratic fields, Invent. Math. 88 (1987), 31-38.

[10] H. Ichimura, On the class groups of pure function fields, Proc. Japan Acad. 64 (1988), 170-173; corrigendum, ibid. 75 (1999), 22. 
[11] K. Iw as awa, A note on class numbers of algebraic number fields, Abh. Math. Sem. Univ. Hamburg 20 (1956), 257-258.

[12] I. Kimura, On class numbers of quadratic extensions over function fields, Manuscripta Math. 97 (1998), 81-91.

[13] T. Nagell, Über die Klassenzahl imaginär-quadratischer Zahlkörper, Abh. Math. Sem. Univ. Hamburg 1 (1922), 140-150.

[14] P. Roquette and H. Zassenhaus, A class rank estimate for algebraic number fields, J. London Math. Soc. 44 (1969), 31-38.

[15] M. Rosen, The Hilbert class fields in function fields, Exposition. Math. 5 (1987), 365-378.

[16] D. Shanks, The simplest cubic fields, Math. Comp. 28 (1974), 1137-1157.

[17] Y. Yamamoto, On unramified Galois extensions of quadratic number fields, Osaka J. Math. 7 (1970), 57-76.

Department of Mathematics

Yokohama City University

22-2, Seto, Kanazawa-ku

Yokohama, 236-0027 Japan

E-mail: ichimura@yokohama-cu.ac.jp 